\title{
Research Article \\ Robust Tracking Control for Switched Fuzzy Systems with Fast Switching Controller
}

\author{
Hong Yang, ${ }^{1}$ Le Zhang, ${ }^{1}$ and Xiaodong Liu ${ }^{2}$ \\ ${ }^{1}$ School of Information Engineering, Shenyang University, Shenyang 110044, China \\ ${ }^{2}$ School of Electronic and Information Engineering, Dalian University of Technology, Dalian 116023, China \\ Correspondence should be addressed to Hong Yang, cherryyh@126.com
}

Received 30 August 2012; Revised 27 October 2012; Accepted 12 November 2012

Academic Editor: Wudhichai Assawinchaichote

Copyright (C) 2012 Hong Yang et al. This is an open access article distributed under the Creative Commons Attribution License, which permits unrestricted use, distribution, and reproduction in any medium, provided the original work is properly cited.

This paper addresses the problem of designing robust tracking controls for a class of switched fuzzy (SF) systems with time delay. A switched fuzzy system, which differs from existing ones, is firstly employed to describe a nonlinear system. Next, a fast switching controller consisting of a number of simple subcontrollers is proposed. The smooth transition is governed by using the fast switching controller. Tracking hybrid control schemes which are based upon a combination of the $H_{\infty}$ tracking theory, fast switching control algorithm, and switching law design are developed such that the $H_{\infty}$ model referent tracking performance is guaranteed. Since convex combination techniques are used to derive the delay independent criteria, some subsystems are allowed to be unstable. Finally, various comparisons of the elaborated examples are conducted to demonstrate the effectiveness of the proposed control design approach. All results illustrate good control performances as desired.

\section{Introduction}

Recent years have witnessed rapidly growing interest in switched systems which are an important class of hybrid systems [1-6]. Time delays, due to the information transmission between subsystems, naturally exist in switched systems, and the existence of the delay is frequently a source of instability. So, much research has been devoted to the study of switched systems with time delay [7-11]. Since switched systems with time-delay have a strong engineering background, special attention has been attracted, and several useful results have been reported in the literature such as the issues on stability analysis [12-15], $H_{\infty}$ filtering [16], and tracking control [17].

From the middle of the 1980s, there have appeared a number of analysis problems about T-S fuzzy systems [18-28], and recently, switched systems have been extended further 
to encompass fuzzy systems too. Very notably, the stabilizability conditions and smoothness conditions for fuzzy switching control systems were reported. For the continuous-time case, a combination of hybrid systems and fuzzy multiple model systems was described, and an idea of the fuzzy switched hybrid control was put forward [20]. Based on the T-S fuzzy systems, Tanaka et al. [21-24] introduced new switching fuzzy systems for more complicated real systems such as multiple nonlinear systems, switched nonlinear hybrid systems, and secondorder nonholomonic systems. Tanaka et al. also addressed why the switching fuzzy model not the T-S fuzzy model is needed with a practical example in [21-23]. For the discrete-time case, Choi gave some extensions in [21-23] to output control [25] and to guaranteed cost [26] control designs. This class of the model [21-23] has two levels of structures. It will switch between the second level region rules according to the first level region rules. In fact, it is a type of switching for the same premise variable.

To effectively achieve nonlinear control, we propose the SF model [27]. The proposed corresponding model of SF systems, presented below, differs from the existing ones in the literature cited in the fact that each sub system is a T-S fuzzy system hence defining a class of SF systems. Usually, this class of systems can precisely describe continuous and discrete dynamics as well as their interactions in the complex real-world systems. It should be noted this class inherits some essential features of hybrid systems [29] while retaining all the information and knowledge representation capacity of fuzzy systems [30].

Comparing with the previous work [21-23], this model of SF system has not two levels of structures, but it is switching between each of the subfuzzy systems directly, not depending on region fuzzy rules. That is to say it is extended further for the switching fuzzy systems. Furthermore, this model is used for the extra design of the switching law based on the previous work [21-23]. In the case, stability analysis is often facilitated by the fact that properties of each individual subfuzzy system are of concern only in the regions where this subsystem is active, and the behavior of the sub fuzzy systems in other parts of the state space has no influence on the SF system. It is possible to find a switching law that renders the SF systems stable for instable sub fuzzy systems. Also, switching law can be designed by arbitrary form, not only depending on fuzzy rules as [21-23]. To the authors' knowledge, the issue of tracking control, which has been well addressed for switched systems with delay $[17,31]$ and fuzzy systems with delay [32-34], has been rarely investigated for SF systems with time-delay. The recent paper [35] has investigated the adaptive robust tracking control of SF systems; however, time delays are not considered. Tracking control for SF systems with time delay which is based on designed switching method is still an open and interesting issue.

On the other hand, traditional parallel distributed compensation (PDC) control [21-23, 36] is good at handling switching fuzzy systems subject. However, an undesired chattering effect will occur. Throughout this paper, the fast switching controller, which consists of a number of simple subcontrollers, is designed from sub fuzzy system of SF model. One of the sub-controllers will be active at a time according to some switching laws derived from the Lyapunov stability theory. Consequently, a fast switching controller, which provides good system performance and has no chattering effect when the system state approaches the origin, can be obtained. Furthermore, a switching function is employed to alleviate the chattering effect during the transient period.

In this paper, we investigate the problem of tracking control for SF systems with time delay by using a switching method. Sufficient conditions for the solvability of the tracking control problem are given. We use single Lyapunov function technique and the fast switching controller to design a tracking control law, such that the $H_{\infty}$ model reference tracking performance is satisfied. Since convex combination techniques are used to derive the delay 
independent criteria, some subsystems are allowed to be unstable. It is highly desirable that a nonswitched time-delay system cannot earn such property. Finally, the simulation example shows the validity of the proposed methods.

This paper is organized as follows. Section 2 reviews a switching fuzzy system. In Section 3, we describe the model of a SF system of time-delay case. In Section 4, new stabilization and the fast switching controller are derived. Two compared example simulations will be presented in Section 5. Finally, a conclusion will be drawn in Section 6.

\section{Preliminaries}

Consider a switching fuzzy model defined by Tanaka et al. as follows [21-23]: region rule $j$ :

$$
\text { if } z_{1}(t) \text { is } N_{j 1} \text { and } \ldots \text { and } z_{p}(t) \text { is } N_{j p}
$$

then

local region rule $i$ :

$$
\begin{aligned}
& \text { if } z_{1}(t) \text { is } M_{j i 1} \text { and } \ldots \text { and } z_{p}(t) \text { is } M_{j i p} \\
& \text { then } \\
& \dot{x}(t)=A_{j i} x(t)+B_{j i} u(t), \\
& y(t)=C_{j i} x(t), \\
& i=1,2, \ldots, r, \quad j=1,2, \ldots, s .
\end{aligned}
$$

$z_{1}(t), \ldots, z_{p}(t)$ are the premise variables. $M_{j i 1}, \ldots, M_{j i p}$ are the fuzzy sets. $r$ is the number of the if-then rules. This switching fuzzy model in [21-23] has two levels of structures: region rule level and local fuzzy rule level. The region rule is crisply switched according to the premise variables. $s$ is the number of region partitioned on the premise variables space. $N_{j k}(z(t))$ is a crisp set.

Where

$$
N_{j k}(z(t))= \begin{cases}1, & z(t) \in N_{j k} \\ 0, & \text { otherwise }\end{cases}
$$

The following final output of the switching fuzzy model (2.2) is inferred by Tanaka et al. in [21-23]:

$$
\begin{gathered}
\dot{x}(t)=\sum_{j=1}^{s} \sum_{i=1}^{r} v_{j}(z(t)) h_{j i}(z(t))\left\{A_{j i} x(t)+B_{j i} u(t)\right\}, \\
y(t)=\sum_{j=1}^{s} \sum_{i=1}^{r} v_{j}(z(t)) h_{j i}(z(t)) C_{j i} x(t),
\end{gathered}
$$


where

$$
\begin{gathered}
v_{j}(z(t))=\frac{\prod_{k=1}^{p} N_{j k}\left(z_{k}(t)\right)}{\sum_{j=1}^{s} \prod_{k=1}^{p} N_{j k}\left(z_{k}(t)\right)}, \quad h_{j i}(z(t))=\frac{\prod_{k=1}^{p} M_{j i k}\left(z_{k}(t)\right)}{\sum_{i=1}^{r} \prod_{k=1}^{p} M_{j i k}\left(z_{k}(t)\right)}, \\
v_{j}(z(t))= \begin{cases}1, & z(t) \in \text { Region } j \\
0, & \text { otherwise },\end{cases} \\
\text { Region } 1 \cup \text { Region } 2 \cup \cdots \text { Region } S=\bigcup_{j=1}^{\mathrm{s}} \text { Region } j=X, \\
\text { Region } j_{1} \cap \text { Region } j_{2}=\phi, \\
j_{1} \neq j_{2}, \quad j_{1}=1,2, \ldots, s, \quad j_{2}=1,2, \ldots, s,
\end{gathered}
$$

where $X$ denotes the universe of discourse. When the variables $z_{1}(t), \ldots, z_{p}(t)$ satisfy the condition of the region rule, the fuzzy model which belongs to the local region rule below the region rule is active.

The idea of Tanaka et al. is simple and natural. They explain the idea through the following example [21].

Example 2.1. Consider a switching fuzzy model with the membership functions in Regions 1 and 2 as shown in Figure 1.

then

Region rule 1: if $x_{2}(t) \geq 1$

local region rule 1: if $x_{2}(t)$ is $h_{11}\left(x_{2}(t)\right)$

then $\dot{x}(t)=A_{11} x(t)+B_{11} u(t)$

local region rule 2: if $x_{2}(t)$ is $h_{12}\left(x_{2}(t)\right)$

then $\dot{x}(t)=A_{12} x(t)+B_{12} u(t)$

Region rule 2: if $x_{2}(t)<1$

then

local region rule 1: if $x_{2}(t)$ is $h_{21}\left(x_{2}(t)\right)$

then $\dot{x}(t)=A_{21} x(t)+B_{21} u(t)$.

local region rule 2: if $x_{2}(t)$ is $h_{22}\left(x_{2}(t)\right)$

then $\dot{x}(t)=A_{22} x(t)+B_{22} u(t)$. 


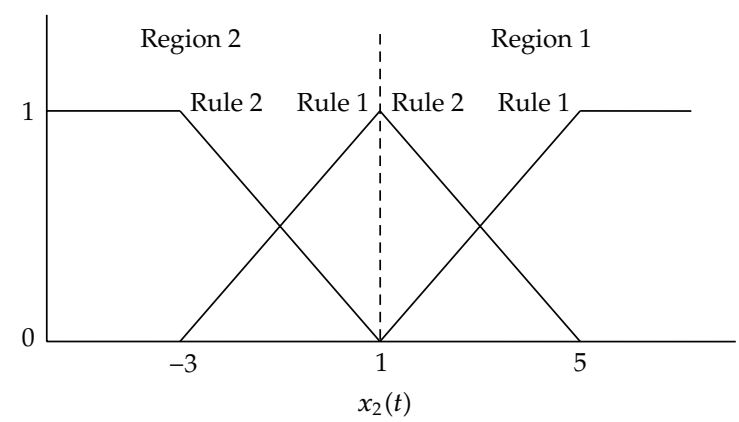

Figure 1: Membership functions of switching fuzzy systems in regions 1 and 2.

The membership functions of each local T-S fuzzy model are assigned as follows:

$$
\begin{aligned}
& h_{11}\left(x_{2}(t)\right)= \begin{cases}\frac{x_{2}(t)-1}{4}, & 1<x_{2}(t) \leq 5 \\
1, & x_{2}(t)>5,\end{cases} \\
& h_{12}\left(x_{2}(t)\right)= \begin{cases}\frac{-x_{2}(t)+5}{4}, & 1<x_{2}(t) \leq 5 \\
0, & x_{2}(t)>5,\end{cases} \\
& h_{21}\left(x_{2}(t)\right)= \begin{cases}\frac{x_{2}(t)+3}{4}, & -3<x_{2}(t) \leq 1 \\
0, & x_{2}(t)<-3,\end{cases} \\
& h_{22}\left(x_{2}(t)\right)= \begin{cases}\frac{-x_{2}(t)+1}{4} & -3 \leq x_{2}(t)<1 \\
1, & x_{2}(t)<-3 .\end{cases}
\end{aligned}
$$

It can be seen from Figure 1 that the connecting rules that bridge between region 1 and region 2 are rule 2 in region 1 and rule 1 in region 2.

Differing from the existing switching fuzzy systems [21-24], we propose the SF model [27]. Now we introduce the SF systems.

When subsystems of the switched systems are T-S fuzzy systems, the systems are switched fuzzy systems. Sketch map of the switched fuzzy systems is depicted in Figure 2. $\Omega_{i}$ denote the state area of the $i$ th switched subsystem. $\Omega_{i l}$ denotes the $l$ th subfuzzy area in $\Omega_{i}$. In fact, the switched fuzzy systems again partition the $\Omega_{i}$ subarea into $l$ subfuzzy areas $\Omega_{i 1}, \ldots, \Omega_{i l}, \ldots, \Omega_{i \ell}$. There is local linear model in every subfuzzy area, namely, local linear model in $\Omega_{i l}$ is $\dot{x}(t)=A_{i l} x(t)+B_{i l} u_{i}(t)$. The model of every switched subarea $\Omega_{1}, \ldots, \Omega_{m}$ is composed of local linear model which is linked by fuzzy membership function. We design the switching law for sub fuzzy area model to ensure stability of the switched fuzzy system. When local model in subfuzzy area satisfies the switching law, we switch to the $\Omega_{i}$ th sub system to ensure stability of the switched fuzzy system.

Now, we define SF model including $N_{\sigma(t)}$ pieces of rules as follows:

$$
R_{\sigma(t)}^{l} \text { : if } \bar{x}_{\sigma(t) 1}(t) \text { is } M_{\sigma(t) 1}^{l} \ldots \text { and } \bar{x}_{\sigma(t) p}(t) \text { is } M_{\sigma(t) p}^{l}
$$




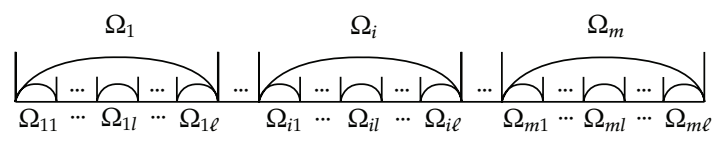

Figure 2: Sketch map of switched fuzzy system.

then

$$
\dot{x}(t)=A_{\sigma(t) l} x(t)+B_{\sigma(t) l} u_{\sigma(t)}(t), \quad l=1,2, \ldots, N_{\sigma(t)},
$$

with

$$
\sigma(t): M=\{1,2, \ldots, m\},
$$

where is a piecewise constant function, called a switching signal.

$R_{\sigma(t)}^{l}$ denotes the lth fuzzy inference rule, $N_{\sigma(t)}$ are the number of inference rules, $u_{\sigma(t)}(t)$ is the input variable, $x(t)$ is the state variable vector, $A_{\sigma(t) l}$ and $B_{\sigma(t) l}$ are matrices of appropriate dimensions, $\bar{x}_{\sigma(t) 1}, \bar{x}_{\sigma(t) 2}, \ldots, \bar{x}_{\sigma(t) p}$ are the vector of premise variables.

Comparing with the previous work [21-23], the SF model breaks through two levels of structures, but it is switching between each of the sub fuzzy model directly. Particularly, when the parameters sudden change or discontinuous change, the switching rules can be designed as any combinational function of variables, which make up the insufficiency of the switching depending on the single variable of the switching fuzzy model. That is, the SF systems are extended further for the switching fuzzy systems (2.2)-(2.4).

\section{Novel Models of Switched Fuzzy Time-Delay Systems}

So in here, we shall introduce an innovated representation modeling of SF time-delay systems. In this model, each subsystem is a fuzzy time-delay system, namely, sub fuzzy timedelay system.

Consider the SF time-delay model including $N_{\sigma(t)}$ pieces of rules as follows:

$$
\begin{gathered}
R_{\sigma(t)}^{l} \text { : if } \bar{x}_{\sigma(t) 1}(t) \text { is } M_{\sigma(t) 1}^{l} \ldots \text { and } \bar{x}_{\sigma(t) p}(t) \text { is } M_{\sigma(t) p^{\prime}}^{l} \\
\text { then } \dot{x}(t)=A_{\sigma(t) l} x(t)+D_{\sigma(t) l} x(t-d)+B_{\sigma(t) l} u_{\sigma(t) l}(t)+\omega, \quad l=1,2, \ldots, N_{\sigma(t)}, \\
y(t)=C_{\sigma(t) l} x(t), \quad x(t)=\varphi(t), \quad t \in[-\tau, 0]
\end{gathered}
$$

with

$$
\sigma(t): M=\{1,2, \ldots, m\},
$$

where is a piecewise constant function, called a switching signal.

$u_{\sigma(t) l}(t) \in \mathbb{R}^{q}$ is the control input, $x(t) \in \mathbb{R}^{n}$ and $y(t) \in \mathbb{R}^{s}$ denote the state vector and the output vector, respectively, $\omega \in \mathbb{R}^{n}$ is the bounded exogenous disturbance, $A_{\sigma(t) l}$ and 
$D_{\sigma(t) l} \in \mathbb{R}^{n \times n}$ are known system matrices, and $B_{\sigma(t) l} \in \mathbb{R}^{n \times q}$ is the input matrix. $C_{\sigma(t) l} \in \mathbb{R}^{s \times n}$ is the output matrix, $d$ is the constant bounded time delay in the state and it is assumed to be $0<d \leq \tau$, and the initial condition $\varphi(t)$ is a differentiable function or constant vector.

It is readily seen that the $i$ th sub fuzzy system can be represented as follows:

$$
R_{i}^{l}: \text { if } \bar{x}_{i 1}(t) \text { is } M_{i 1}^{l} \ldots \text { and } \bar{x}_{i p}(t) \text { is } M_{i p}^{l}
$$

then

$$
\dot{x}(t)=A_{i l} x(t)+D_{i l} x(t-d)+B_{i l} u_{i l}(t)+\omega, \quad l=1,2, \ldots, N_{i}, i=1,2, \ldots, m .
$$

Therefore the global model of the $i$ th sub fuzzy system is described by means of the equation as follows:

$$
\dot{x}(t)=\sum_{l=1}^{N_{i}} \eta_{i l}\left(\bar{x}_{i}(t)\right)\left[A_{i l} x(t)+D_{i l} x(t-d)+B_{i l} u_{i l}(t)+\omega\right],
$$

along with

$$
\begin{array}{cl}
0 \leq \eta_{i l}\left(\bar{x}_{i}(t)\right) \leq 1, & \sum_{l=1}^{N_{i}} \eta_{i l}\left(\bar{x}_{i}(t)\right)=1, \\
w_{i l}\left(\bar{x}_{i}(t)\right)=\prod_{\rho=1}^{p} M_{i \rho}^{l}\left(\bar{x}_{i \rho}(t)\right), & \eta_{i l}\left(\bar{x}_{i}(t)\right)=\frac{w_{i l}\left(\bar{x}_{i}(t)\right)}{\sum_{l=1}^{N_{i}} w_{i l}\left(\bar{x}_{i}(t)\right)},
\end{array}
$$

where $M_{i \rho}^{l}\left(\bar{x}_{i \rho}(t)\right)$ denotes the membership function which $\bar{x}_{i \rho}(t)$ belongs to the fuzzy set $M_{i \rho}^{l}$.

\section{Design of Fast Switching Controllers and Switching Law}

Given a reference model

$$
\dot{x}_{r}(t)=A_{r} x_{r}(t)+r(t), \quad x_{r}(0)=0
$$

and performance index

$$
\int_{0}^{t_{f}} e_{r}^{T}(t) e_{r}(t) d t<r^{2} \int_{0}^{t_{f}} \tilde{\omega}^{T}(t) \tilde{\omega}(t) d t
$$

where $x_{r}(t) \in \mathbb{R}^{n}$ is reference state, $A_{r}$ is a Hurwitz matrix, $r(t)$ is bounded reference input, $e_{r}(t)=x(t)-x_{r}(t)$ denotes the error between the real state of the switched fuzzy time-delay system (3.1) and the reference state, $t_{f}$ is the control terminated time, $(\tilde{\omega}(t)=$ $\left.\left(\omega^{T}(t)-r^{T}(t) r^{T}(t)\right)\right)^{T}$, and $\gamma>0$ is a prescribed attenuation level. 
Definition 4.1. The system (3.1) is said to be quadratically stable if there exists positive definite matrix $P$ and state-dependent switching law $\sigma=\sigma(x)$ such that the quadratic Lyapunov function $V(x(t))$ satisfies $\dot{V}(x(t))<0$ for any $x(t) \neq 0$ along the system state trajectory from arbitrary initial conditions.

It should be noted, in this definition the existence of state is dependent on switching law $\sigma=\sigma(x)$ and how to construct it. It is therefore that it can be pursued the way it is most appropriate for the given class of plant system and the investigated control synthesis so as to obtain the desirable design, preferably the one guaranteeing global asymptotic or exponential stability [1-5]. It is this fact that has been exploited in the sequel in conjunction with the choice of the switching laws in Theorems 4.3.

In here, for system (3.1), if there exist control input $u=u(t)$ and switching signal $\sigma=\sigma(t)$ such that (3.1) is quadratically stable when $\widetilde{\omega} \equiv 0$, and (4.2) is satisfied when $\widetilde{\omega} \neq 0$ under the initial conditions stated in (3.1) and (4.1), then the switched system (3.1) is said to have $H_{\infty}$ model reference tracking performance.

Our purpose is to design a controller $u=u(t)$ and a switching law such that system (3.1) has the $H_{\infty}$ model reference tracking performance.

Remark 4.2. The $H_{\infty}$ tracking control problem for a switched fuzzy time-delay system is solved by Theorems 4.3-4.6. When $M=\{1\}$, switched fuzzy time-delay system (3.1) degenerates into a general fuzzy system, and the $H_{\infty}$ tracking control problem becomes the standard $H_{\infty}$ tracking control problem for fuzzy systems.

Now a fast switching controller is employed to control the switched fuzzy time-delay model of (3.1). The fast switching controller consists of some simple subcontrollers. These sub-controllers will switch among each other to control the system of (3.1) according to an appropriate switching scheme. It is shown in the sequel how to design controllers to achieve quadratic stability in the closed loop and under the switching law.

The fast switching controller for the sub fuzzy time-delay system is described by

$$
u_{i l}(t)=-\sum_{a=1}^{N_{i}} \rho_{i l a}\left(e_{r}(t)\right) R B_{i a}^{T} P_{11} e_{r}(t),
$$

where $\rho_{\text {ila }}\left(e_{r}(t)\right)$ takes the value of 0 or 1 according to a switching scheme discussed later, $R \in$ $\mathbb{R}^{q \times q}$ and $P_{11} \in \mathbb{R}^{n \times n}$ are symmetric positive definite matrices to be designed, and $(\cdot)^{T}$ denotes the transpose of a matrix or a vector.

Combining (3.1) with (4.1) and (4.3), we get the augmented system as follows:

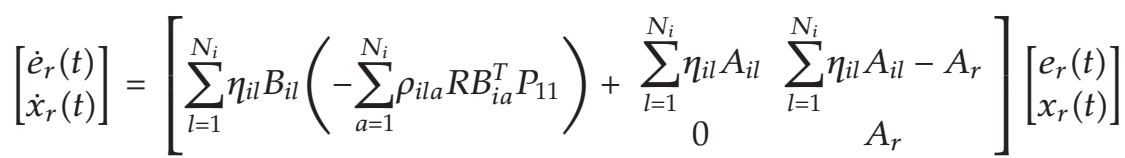

$$
\begin{aligned}
& +\left[\begin{array}{cc}
\sum_{l=1}^{N_{i}} \eta_{i l} D_{i l} & \sum_{l=1}^{N_{i}} \eta_{i l} D_{i l} \\
0 & 0
\end{array}\right]\left[\begin{array}{c}
e_{r}(t-\tau) \\
x_{r}(t-\tau)
\end{array}\right]+\left[\begin{array}{c}
\sum_{l=1}^{N_{i}} \eta_{i l} \omega-r \\
r
\end{array}\right] .
\end{aligned}
$$


Thus, the system (4.4) can be rewritten as

$$
\dot{\tilde{x}}(t)=\sum_{l=1}^{N_{i}} \eta_{i l} \tilde{A}_{i l} \tilde{x}(t)+\sum_{l=1}^{N_{i}} \eta_{i l} \tilde{D}_{i l} \tilde{x}(t-\tau)+\sum_{l=1}^{N_{i}} \eta_{i l} \tilde{\omega}(t)
$$

where

$$
\begin{gathered}
\tilde{x}(t)=\left[\begin{array}{l}
e_{r}(t) \\
x_{r}(t)
\end{array}\right], \quad \tilde{A}_{i l}=\left[\begin{array}{cc}
B_{i l}\left(-\sum_{a=1}^{N_{i}} \rho_{i l a} R B_{i a}^{T} P_{11}\right)+A_{i l} & A_{i l}-A_{r} \\
0 & A_{r}
\end{array}\right], \\
\tilde{D}_{i l}=\left[\begin{array}{cc}
D_{i l} & D_{i l} \\
0 & 0
\end{array}\right], \quad \tilde{\omega}=\left[\begin{array}{c}
\omega-r \\
r
\end{array}\right] .
\end{gathered}
$$

We have the following result.

Theorem 4.3. Suppose there exist positive definite symmetric matrixes $P, S, Q$, and constants $\lambda_{i j_{i}}>0$ such that the following matrix inequalities are satisfied; then for a prescribed $\gamma^{2}, H_{\infty}$ tracking control performance in (4.2) is guaranteed via the state-feedback controller (4.3) as follows:

$$
\sum_{i=1}^{m} \lambda_{i j_{i}}\left[\begin{array}{ccc}
\tilde{A}_{i j_{i}}^{T} P+P \tilde{A}_{i j_{i}}+S+Q & P \tilde{D}_{i j_{i}} & P \\
* & -S & 0 \\
* & * & -\gamma^{2} I
\end{array}\right]<0, \quad j_{i}=1,2 \ldots, N_{i}, i=1,2, \ldots, m
$$

And, the switching law is designed as

$$
\begin{gathered}
\sigma(t)=\arg \min \left\{\overline { V } _ { i } ( t ) \triangleq \operatorname { m a x } _ { j _ { i } } \left\{\Xi^{T}(t)\left[\begin{array}{cc}
\tilde{A}_{i j_{i}}^{T} P+P \tilde{A}_{i j_{i}}+S+Q+\gamma^{-2} P P & P \widetilde{D}_{i j_{i}} \\
\tilde{D}_{i j_{i}}^{T} P & -S
\end{array}\right] \Xi(t)<0,\right.\right. \\
\left.\left.j_{i}=1,2, \ldots, N_{i}\right\}\right\} .
\end{gathered}
$$

Proof. By Schur complement lemma, the condition (4.7) is equivalent to the following inequality:

$$
\sum_{i=1}^{m} \lambda_{i j_{i}}\left[\begin{array}{cc}
\tilde{A}_{i j_{i}}^{T} P+P \tilde{A}_{i j_{i}}+S+Q+\gamma^{-2} P P & P \tilde{D}_{i j_{i}} \\
\tilde{D}_{i j_{i}}^{T} P & -S
\end{array}\right]<0
$$

From (4.9) we know that for any $\Xi(t) \neq 0$, it holds that

$$
\sum_{i=1}^{m} \lambda_{i j_{i}} \Xi^{T}(t)\left[\begin{array}{cc}
\tilde{A}_{i j_{i}}^{T} P+P \tilde{A}_{i j_{i}}+S+Q+\gamma^{-2} P P & P \tilde{D}_{i j_{i}} \\
\tilde{D}_{i j_{i}}^{T} P & -S
\end{array}\right] \Xi(t)<0 .
$$


Note that (4.10) holds for any $j_{i} \in\left\{1,2, \ldots, N_{i}\right\}$ and $\lambda_{i j_{i}}>0$, then there exists at least an $i$ such that for any $j_{i}$

$$
\Xi^{T}(t)\left[\begin{array}{cc}
\tilde{A}_{i j_{i}}^{T} P+P \tilde{A}_{i j_{i}}+S+Q+\gamma^{-2} P P & P \widetilde{D}_{i j_{i}} \\
\tilde{D}_{i j_{i}}^{T} P & -S
\end{array}\right] \Xi(t)<0 .
$$

Thus, the switching law defined by (4.8) is well defined.

Now define a quadratic Lyapunov-Krasovskii functional candidate

$$
V(\tilde{x}(t))=\tilde{x}^{T}(t) P \tilde{x}(t)+\int_{t-\tau}^{t} \tilde{x}^{T}(\theta) S \tilde{x}(\theta) d \theta
$$

which is positive definite, since $P$ and $S$ are positive definite matrices.

First, we will prove that the system (4.5) is quadratically stable while $\widetilde{\omega}(t) \equiv 0$. The time derivative of $V(t)$ is

$$
\begin{aligned}
\dot{V}(\tilde{x}(t))= & \dot{\tilde{x}}^{T}(t) P \tilde{x}(t)+\tilde{x}^{T}(t) P \dot{\tilde{x}}(t)+\tilde{x}^{T}(t) S \tilde{x}(t)-\tilde{x}^{T}(t-\tau) S \tilde{x}(t-\tau) \\
= & \sum_{l=1}^{N_{i}} \eta_{i l} \tilde{x}^{T}(t)\left(\tilde{A}_{i l}^{T} P+P \tilde{A}_{i l}\right) \tilde{x}(t)+\sum_{l=1}^{N_{i}} \eta_{i l} \tilde{x}^{T}(t) P \tilde{D}_{i l} \tilde{x}(t-\tau)+\sum_{l=1}^{N_{i}} \eta_{i l} \tilde{x}^{T}(t-\tau) \tilde{D}_{i l}^{T} P \tilde{x}(t) \\
& +\tilde{x}^{T}(t) S \tilde{x}(t)-\tilde{x}^{T}(t-\tau) S \tilde{x}(t-\tau) \\
= & \sum_{l=1}^{N_{i}} \eta_{i l} \Xi^{T}(t)\left[\begin{array}{cc}
\tilde{A}_{i l}^{T} P+P \tilde{A}_{i l}+S & P \tilde{D}_{i l} \\
\tilde{D}_{i l}^{T} P & -S
\end{array}\right] \Xi(t),
\end{aligned}
$$

where $\Xi(t)=\left[\begin{array}{c}\tilde{x}(t) \\ \tilde{x}(t-\tau)\end{array}\right]$.

Then from (4.11), it holds true that

$$
\begin{aligned}
& \Xi^{T}(t)\left[\begin{array}{cc}
\tilde{A}_{i j_{i}}^{T} P+P \tilde{A}_{i j_{i}}+S+Q+\gamma^{-2} P P & P \widetilde{D}_{i j_{i}} \\
\tilde{D}_{i j_{i}}^{T} P & -S
\end{array}\right] \Xi(t)
\end{aligned}
$$

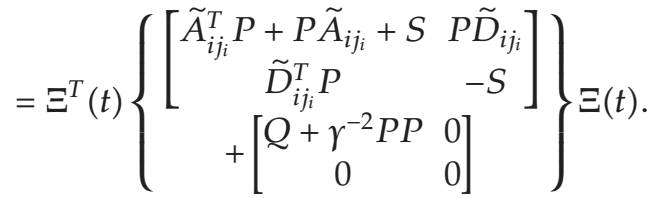

$$
\begin{aligned}
& <0
\end{aligned}
$$

in which $i=\sigma(t)$ given by (4.8). Taking (3.6), (4.11), and (4.14) into account we deduce that $(d / d t) V(\tilde{x}(t))<0, \Xi(t) \neq 0$. Therefore, system (4.5) is quadratically stable under the switching law (4.8). 
Next, under the zero initial condition we prove

$$
\int_{0}^{t_{f}} e_{r}^{T}(t) e_{r}(t) d t<r^{2} \int_{0}^{t_{f}} \tilde{\omega}^{T}(t) \tilde{\omega}(t) d t \quad \text { when } \tilde{\omega}(t) \neq 0
$$

Differentiating the Lyapunov-Krasovskii functional candidate along the trajectories $\tilde{x}(t)$ of the system (4.5) gives

$$
\dot{V}(\tilde{x}(t))=\sum_{l=1}^{N_{i}} \eta_{i l} \Xi^{T}(t)\left[\begin{array}{cc}
\tilde{A}_{i l}^{T} P+P \tilde{A}_{i l}+S & P \widetilde{D}_{i l} \\
\tilde{D}_{i l}^{T} P & -S
\end{array}\right] \Xi(t)+2 \sum_{l=1}^{N_{i}} \eta_{i l} \tilde{x}^{T}(t) P \tilde{\omega}(t) .
$$

So we get

$$
2 \tilde{x}^{T}(t) P \tilde{\omega}(t) \leq \gamma^{-2} \tilde{x}^{T}(t) P P \tilde{x}(t)+\gamma^{2} \tilde{\omega}^{T}(t) \tilde{\omega}(t)
$$

then

$$
\dot{V}(\tilde{x}(t))=\sum_{l=1}^{N_{i}} \eta_{i l} \Xi^{T}(t)\left[\begin{array}{cc}
\tilde{A}_{i l}^{T} P+P \tilde{A}_{i l}+S+\gamma^{-2} P P & P \tilde{D}_{i l} \\
\tilde{D}_{i l}^{T} P & -S
\end{array}\right] \Xi(t)+\sum_{l=1}^{N_{i}} \eta_{i l} \gamma^{2} \tilde{\omega}^{T}(t) \tilde{\omega}(t) .
$$

By the switching law (4.11), when the $i$ th subsystem is active, it gives that

$$
\Xi^{T}(t)\left[\begin{array}{cc}
\tilde{A}_{i l}^{T} P+P \tilde{A}_{i l}+S+\gamma^{-2} P P & P \widetilde{D}_{i l} \\
\widetilde{D}_{i l}^{T} P & -S
\end{array}\right] \Xi(t)<\Xi^{T}(t)\left[\begin{array}{cc}
-Q & 0 \\
0 & 0
\end{array}\right] \Xi(t) .
$$

Substituting (4.19) into (4.18), we obtain

$$
\dot{V}(\tilde{x}(t))<-\sum_{l=1}^{N_{i}} \eta_{i l} \tilde{x}^{T}(t) Q \tilde{x}(t)+\sum_{l=1}^{N_{i}} \eta_{i l} r^{2} \tilde{\omega}^{T}(t) \tilde{\omega}(t),
$$

where

$$
\tilde{x}^{T}(t) Q \tilde{x}(t)=\left[\begin{array}{l}
e_{r}(t) \\
x_{r}(t)
\end{array}\right]^{T}\left[\begin{array}{ll}
I & 0 \\
0 & 0
\end{array}\right]\left[\begin{array}{l}
e_{r}(t) \\
x_{r}(t)
\end{array}\right]=e_{r}^{T}(t) e_{r}(t)
$$

Substituting (4.21) into (4.20) results in

$$
\dot{V}(\tilde{x}(t))<-\sum_{l=1}^{N_{i}} \eta_{i l} e_{r}^{T}(t) e_{r}(t)+\sum_{l=1}^{N_{i}} \eta_{i l} r^{2} \tilde{\omega}^{T}(t) \tilde{\omega}(t) .
$$

It is easy to derive [37]

$$
\int_{0}^{t_{f}} e_{r}^{T}(t) e_{r}(t) d t<r^{2} \int_{0}^{t_{f}} \tilde{\omega}^{T}(t) \tilde{\omega}(t) d t
$$

That is (4.2), and the $H_{\infty}$ control performance is achieved with a prescribed $\gamma^{2}$. 
It is noted that Theorem 4.3 gives the sufficient condition of ensuring the stability of the SF time-delay system (3.1) and achieving the $H_{\infty}$ tracking performance (4.23). However, it does not give the methods of obtaining the solution of a common positive matrix $P$ for (4.7). In general, it is not easy to analytically determine such a common positive matrix, and fortunately (4.7) can be transferred into LMIs, which can be solved in a computationally efficient manner using convex optimization techniques such as the interior point method [38].

For the convenience of the design, we assume

$$
P=\left[\begin{array}{cc}
P_{11} & 0 \\
0 & P_{22}
\end{array}\right], \quad S=\left[\begin{array}{cc}
S_{11} & 0 \\
0 & S_{22}
\end{array}\right] .
$$

We obtain

$$
\begin{aligned}
\Xi^{T}(t)\left[\begin{array}{cc}
\Omega_{i l} & P \tilde{D}_{i l} \\
\tilde{D}_{i l}^{T} P & -S
\end{array}\right] \Xi(t)= & \tilde{x}^{T}(t) \Omega_{i l} \tilde{x}(t)+\tilde{x}^{T}(t-\tau) \tilde{D}_{i l}^{T} P \tilde{x}(t) \\
& +\tilde{x}^{T}(t) P \tilde{D}_{i l} \tilde{x}(t-\tau)+\tilde{x}^{T}(t-\tau)(-S) \tilde{x}(t-\tau),
\end{aligned}
$$

where $\Omega_{i l}=\tilde{A}_{i l}^{T} P+P \tilde{A}_{i l}+S+\gamma^{-2} P P+Q$.

And

$$
\begin{aligned}
& P \tilde{D}_{i l}=\left[\begin{array}{cc}
P_{11} & 0 \\
0 & P_{22}
\end{array}\right]\left[\begin{array}{cc}
D_{i l} & D_{i l} \\
0 & 0
\end{array}\right]=\left[\begin{array}{cc}
P_{11} D_{i l} & P_{11} D_{i l} \\
0 & 0
\end{array}\right], \\
& \tilde{A}_{i l}^{T} P+P \tilde{A}_{i l}=\left[\begin{array}{cc}
\left(-\sum_{a=1}^{N_{i}} \rho_{i l a} R B_{i a}^{T} P_{11}\right)^{T} B_{i l}^{T} P_{11}+A_{i l}^{T} P_{11} & \\
+P_{11} B_{i l}\left(-\sum_{a=1}^{N_{i}} \rho_{i l a} R B_{i a}^{T} P_{11}\right)+P_{11} A_{i l} & P_{11} A_{i l}-P_{11} A_{r} \\
A_{i l}^{T} P_{11}-A_{r}^{T} P_{11} & P_{22} A_{r}+A_{r}^{T} P_{22}
\end{array}\right], \\
& \tilde{D}_{i l}^{T} P=\left[\begin{array}{cc}
D_{i l}^{T} & 0 \\
D_{i l}^{T} & 0
\end{array}\right]\left[\begin{array}{cc}
P_{11} & 0 \\
0 & P_{22}
\end{array}\right]=\left[\begin{array}{cc}
D_{i l}^{T} P_{11} & 0 \\
D_{i l}^{T} P_{11} & 0
\end{array}\right], \\
& \tilde{x}^{T}(t) \Omega_{i l} \tilde{x}(t)=e_{r}^{T}(t) \Lambda_{i l} e_{r}(t)+x_{r}^{T}(t)\left(A_{i l}^{T} P_{11}-A_{r}^{T} P_{11}\right) e_{r}(t)+e_{r}^{T}(t)\left(P_{11} A_{i l}-P_{11} A_{r}\right) x_{r}(t) \\
& +x_{r}^{T}(t)\left(P_{22} A_{r}+A_{r}^{T} P_{22}+S_{22}+\gamma^{-2} P_{22} P_{22}\right) x_{r}(t), \\
& \Lambda_{i l}=\left(-\sum_{a=1}^{N_{i}} \rho_{i l a} R B_{i a}^{T} P_{11}\right)^{T} B_{i l}^{T} P_{11}+A_{i l}^{T} P_{11}+P_{11} B_{i l}\left(-\sum_{a=1}^{N_{i}} \rho_{i l a} R B_{i a}^{T} P_{11}\right) \\
& +P_{11} A_{i l}+S_{11}+\gamma^{-2} P_{11} P_{11}+I \text {. }
\end{aligned}
$$


From (4.27), we have

$$
\begin{aligned}
e_{r}^{T}(t) \Lambda_{i l} e_{r}(t) & \\
=e_{r}^{T}(t)[ & \left(-\sum_{a=1}^{N_{i}} \rho_{i l a} R B_{i a}^{T} P_{11}\right)^{T} B_{i l}^{T} P_{11}+P_{11} B_{i l}\left(-\sum_{a=1}^{N_{i}} \rho_{i l a} R B_{i a}^{T} P_{11}\right) \\
& \left.+A_{i l}^{T} P_{11}+P_{11} A_{i l}+S_{11}+r^{-2} P_{11} P_{11}+I\right] e_{r}(t) \\
=e_{r}^{T}(t)\{ & {\left[\left(-\sum_{a=1}^{N_{i}} \rho_{i l a} R B_{i a}^{T} P_{11}\right)^{T} B_{i l}^{T} P_{11}-\sum_{a=1}^{N_{i}} \eta_{i a}\left(B_{i l} R B_{i a}^{T} P_{11}\right)^{T} P_{11}+\sum_{a=1}^{N_{i}} \eta_{i a}\left(B_{i l} R B_{i a}^{T} P_{11}\right)^{T} P_{11}\right] } \\
& +P_{11} B_{i l}\left(-\sum_{a=1}^{N_{i}} \rho_{i l a} R B_{i a}^{T} P_{11}\right)-\sum_{a=1}^{N_{i}} \eta_{i a} P_{11}\left(B_{i l} R B_{i a}^{T} P_{11}\right)+\sum_{a=1}^{N_{i}} \eta_{i a} P_{11}\left(B_{i l} R B_{i a}^{T} P_{11}\right) \\
& \left.+A_{i l}^{T} P_{11}+P_{11} A_{i l}+S_{11}+\gamma^{-2} P_{11} P_{11}+I\right\} e_{r}(t) \\
=e_{r}^{T}(t)\{ & -\sum_{a=1}^{N_{i}} \eta_{i a}\left(B_{i l} R B_{i a}^{T} P_{11}\right)^{T} P_{11}-\sum_{a=1}^{N_{i}}\left(\rho_{i l a}-\eta_{i a}\right)\left(B_{i l} R B_{i a}^{T} P_{11}\right)^{T} P_{11} \\
& -\sum_{a=1}^{N_{i}} \eta_{i a} P_{11}\left(B_{i l} R B_{i a}^{T} P_{11}\right)-\sum_{a=1}^{N_{i}}\left(\rho_{i l a}-\eta_{i a}\right) P_{11}\left(B_{i l} R B_{i a}^{T} P_{11}\right) \\
+ & \left.A_{i l}^{T} P_{11}+P_{11} A_{i l}+S_{11}+\gamma^{-2} P_{11} P_{11}+I\right\} e_{r}(t) .
\end{aligned}
$$

Let

$$
\begin{gathered}
\rho_{\text {ila }}=\frac{1+\operatorname{sgn}\left(e_{r}^{T}(t) P_{11} B_{i l} R B_{i a}^{T} P_{11} e_{r}(t)\right)}{2}, \\
\operatorname{sgn}(z)= \begin{cases}1 & \text { if } z>0 \\
-1 & \text { otherwise. }\end{cases}
\end{gathered}
$$

From (4.28), we have

$$
\begin{aligned}
& e_{r}^{T}(t) \Lambda_{i l} e_{r}(t) \\
&=e_{r}^{T}(t)\left\{\sum_{a=1}^{N_{i}} \eta_{i a}\left(-P_{11} B_{i a} R B_{i l}^{T} P_{11}-P_{11} B_{i l} R B_{i a}^{T} P_{11}+A_{i l}^{T} P_{11}+P_{11} A_{i l}+S_{11}+\gamma^{-2} P_{11} P_{11}+I\right)\right. \\
&\left.\quad-2 \sum_{a=1}^{N_{i}}\left(\frac{1+\operatorname{sgn}\left(e_{r}^{T}(t) P_{11} B_{i l} R B_{i a}^{T} P_{11} e_{r}(t)\right)}{2}-\frac{1}{2}-\left(\eta_{i a}-\frac{1}{2}\right)\right)\left(B_{i l} R B_{i a}^{T} P_{11}\right)^{T} P_{11}\right\} e_{r}(t)
\end{aligned}
$$




$$
\begin{aligned}
= & e_{r}^{T}(t)\left\{\sum_{a=1}^{N_{i}} \eta_{i a}\left(-P_{11} B_{i a} R B_{i l}^{T} P_{11}-P_{11} B_{i l} R B_{i a}^{T} P_{11}+A_{i l}^{T} P_{11}+P_{11} A_{i l}+S_{11}+\gamma^{-2} P_{11} P_{11}+I\right)\right. \\
& \left.-2 \sum_{a=1}^{N_{i}}\left(\frac{\operatorname{sgn}\left(e_{r}^{T}(t) P_{11} B_{i l} R B_{i a}^{T} P_{11} e_{r}(t)\right)}{2}-\left(\eta_{i a}-\frac{1}{2}\right)\right)\left(B_{i l} R B_{i a}^{T} P_{11}\right)^{T} P_{11}\right\} e_{r}(t) \\
\leq & \sum_{a=1}^{N_{i}} \eta_{i a} e_{r}^{T}(t)\left(-P_{11} B_{i a} R B_{i l}^{T} P_{11}-P_{11} B_{i l} R B_{i a}^{T} P_{11}+A_{i l}^{T} P_{11}+P_{11} A_{i l}+S_{11}+\gamma^{-2} P_{11} P_{11}+I\right) e_{r}(t) \\
& -2 \sum_{a=1}^{N_{i}}\left(\frac{1}{2}-\left(\eta_{i a}-\frac{1}{2}\right)\right)\left|e_{r}^{T}(t) P_{11} B_{i l} R B_{i a}^{T} P_{11} e_{r}(t)\right|
\end{aligned}
$$

As $\eta_{i a}-1 / 2 \in[-1 / 21 / 2]$, due to the property of the switched fuzzy model with time-delay, it can be shown that (4.30) satisfies the following inequality.

$$
\begin{aligned}
& e_{r}^{T}(t) \Lambda_{i l} e_{r}(t) \\
& \quad \leq \sum_{a=1}^{N_{i}} \eta_{i a} e_{r}^{T}(t)\left(-P_{11} B_{i a} R B_{i l}^{T} P_{11}-P_{11} B_{i l} R B_{i a}^{T} P_{11}+A_{i l}^{T} P_{11}+P_{11} A_{i l}+S_{11}+\gamma^{-2} P_{11} P_{11}+I\right) e_{r}(t) .
\end{aligned}
$$

Hence, we can conclude that the closed-loop system of (3.1) is quadratically stable and achieve the $H_{\infty}$ tracking control performance, if the stability condition of (4.32) is satisfied, and the switching scheme of (4.8) is applied. The analysis result is summarized by the Theorem 4.5.

Remark 4.4. It can be seen from (4.3) that the fast switching controller $u_{i l}(t)$ consists of $2^{N_{i}}$ tracking controllers which are linear combinations of $-R B_{i a}^{T} P_{11} e_{r}(t), a=1,2, \ldots, N_{i}$. Taking into account the switching signal, in practice this controller is a hybrid switching-robust controller.

Theorem 4.5. Suppose there exist positive definite matrixes $R, P_{11}, P_{22}, S_{11}, S_{22}$, and constants $\lambda_{i j_{i}}>$ 0 such that the following matrix inequalities are satisfied; then for a prescribed $\gamma^{2}, H_{\infty}$ tracking control performance in (4.2) is guaranteed via the state-feedback controller (4.3), and the switching law is designed as (4.8)

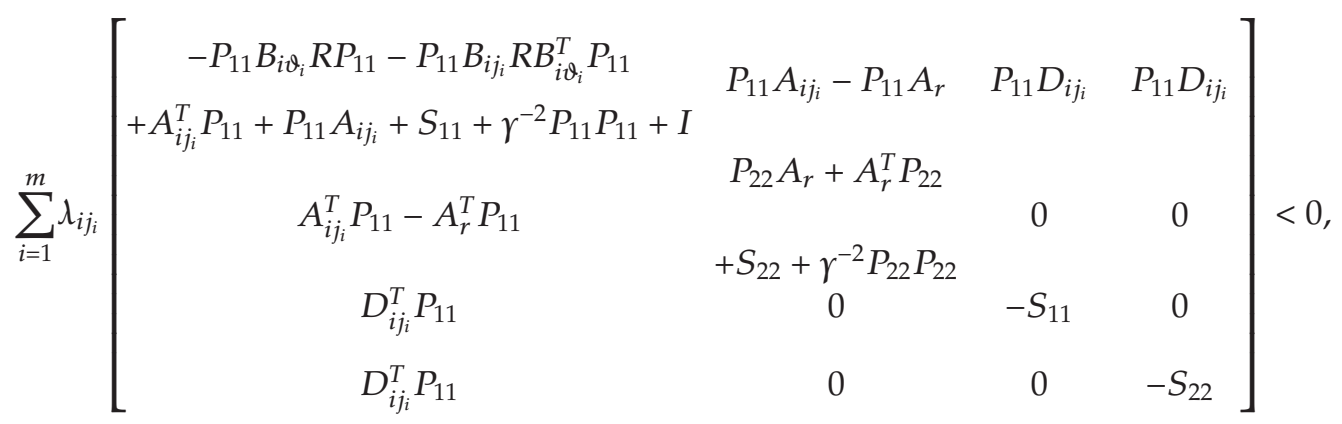

$$
\begin{aligned}
& j_{i}=1,2 \ldots, N_{i}, \quad \vartheta_{i}=1,2 \ldots, N_{i}, \quad i=1,2, \ldots, m .
\end{aligned}
$$


In order words, we formulate the finding of $R$ and $P_{11}$ of (4.32) into LMI problem. Considering (4.32) and multiplying both sides of (4.32) by the matrix $\operatorname{diag}\left\{P_{11}^{-1}, I, I, I\right\}$, we restate as follows.

Theorem 4.6. Suppose there exist positive definite matrixes $R, P_{11}, P_{22}, S_{11}, S_{22}$, and constants $\lambda_{i j_{i}}>$ 0 such that the following matrix inequalities are satisfied; then for a prescribed $\gamma^{2}, H_{\infty}$ tracking control performance in (4.2) is guaranteed via the state-feedback controller (4.3), and the switching law is designed as (4.8)

$$
\sum_{i=1}^{m} \lambda_{i j_{i}}\left[\begin{array}{cccccc}
-B_{i \vartheta_{i}} R B_{i j_{i}}^{T}-B_{i j_{i}} R B_{i \vartheta_{i}}^{T} & A_{i j_{i}}-A_{r} & D_{i j_{i}} & D_{i j_{i}} & P_{11}^{-1} & 0 \\
+P_{11}^{-1} A_{i j_{i}}^{T}+A_{i j_{i}} P_{11}^{-1}+\gamma^{-2} I & P_{22} A_{r}+A_{r}^{T} P_{22}+S_{22} & 0 & 0 & 0 & P_{22} \\
* & * & -S_{11} & 0 & 0 & 0 \\
* & * & * & -S_{22} & 0 & 0 \\
* & * & * & * & -\left(I+S_{11}^{-1}\right) & 0 \\
* & * & * & * & * & -\gamma^{2} I
\end{array}\right]<0,
$$

Once we have $R, P_{11}, P_{22}, S_{11}$, and $S_{22}$ from (4.33), the tracking controllers (4.3) can be constructed.

Remark 4.7. It can be seen that (4.33) is an LMI. $R$ and $P_{11}$ can be solved readily by employing some LMI tools. The analysis results, switching scheme and design of the fast switching controller are summarized by Theorem 4.6.

\section{Illustrative Examples and Results}

In order to demonstrate the efficiency and feasible performance of the proposed control synthesis, now we consider the model as follows:

$$
\begin{aligned}
& R_{1}^{1}: \text { if } x_{2}(t) \text { is } M_{11}^{1} \text {, then } \dot{x}(t)=A_{11} x(t)+D_{11} x(t-d)+B_{11} u_{11}(t)+\omega, \\
& R_{1}^{2}: \text { if } x_{2}(t) \text { is } M_{11}^{2} \text {, then } \dot{x}(t)=A_{12} x(t)+D_{12} x(t-d)+B_{12} u_{12}(t)+\omega, \\
& R_{2}^{1}: \text { if } x_{2}(t) \text { is } M_{21}^{1} \text {, then } \dot{x}(t)=A_{21} x(t)+D_{21} x(t-d)+B_{21} u_{21}(t)+\omega, \\
& R_{2}^{2}: \text { if } x_{2}(t) \text { is } M_{21}^{2} \text {, then } \dot{x}(t)=A_{22} x(t)+D_{22} x(t-d)+B_{22} u_{22}(t)+\omega .
\end{aligned}
$$

Also, this model is used for the extra design of the switching law (4.8) of the statedependent form. For the purpose of comparing with SF system, Example 2.1 in [21] can be described for

region rule 1: if $x_{2}(t) \geq 1$ then

Local Region Rule 1: if $x_{2}(t)$ is $h_{11}\left(x_{2}(t)\right)$

$$
\text { then } \dot{x}(t)=A_{11} x(t)+D_{11} x(t-d)+B_{11} u(t)+\omega \text {, }
$$


Local Region Rule 2: if $x_{2}(t)$ is $h_{12}\left(x_{2}(t)\right)$

$$
\text { then } \dot{x}(t)=A_{12} x(t)+D_{12} x(t-d)+B_{12} u(t)+\omega \text {. }
$$

region rule 2 : if $\mathrm{x}_{2}(t)<1$ then

Local Region Rule 1: if $x_{2}(t)$ is $h_{21}\left(x_{2}(t)\right)$

$$
\text { then } \dot{x}(t)=A_{21} x(t)+D_{21} x(t-d)+B_{21} u(t)+\omega \text {, }
$$

Local Region Rule 2: if $x_{2}(t)$ is $h_{22}\left(x_{2}(t)\right)$

$$
\text { then } \dot{x}(t)=A_{22} x(t)+D_{22} x(t-d)+B_{22} u(t)+\omega \text {. }
$$

Obviously, switching fuzzy system of Example 2.1 is switching between each of the sub fuzzy systems and depending on region fuzzy rules.

We have

$$
\begin{gathered}
A_{11}=\left[\begin{array}{cc}
-10.5 & 5 \\
-0.943 & -1.0493
\end{array}\right], \quad B_{11}=\left[\begin{array}{c}
0.1 \\
-0.4926
\end{array}\right], \quad A_{12}=\left[\begin{array}{cc}
-4.5 & 1 \\
-0.132 & -1.4529
\end{array}\right], \\
B_{12}=\left[\begin{array}{c}
2 \\
-0.1316
\end{array}\right], \quad A_{21}=\left[\begin{array}{cc}
-10 & 0.8 \\
-0.8 & -0.9
\end{array}\right], \quad B_{21}=\left[\begin{array}{cc}
3 \\
-0.01
\end{array}\right], \\
A_{22}=\left[\begin{array}{cc}
-11 & 0.1 \\
-2 & -4.529
\end{array}\right], \quad B_{22}=\left[\begin{array}{c}
1 \\
-0.1765
\end{array}\right], \quad A_{r}=\left[\begin{array}{cc}
-9.8 & 0.02 \\
-0.02 & -4.5
\end{array}\right], \\
D_{11}=\left[\begin{array}{cc}
0.3 & 0.2 \\
0.1 & 0.3
\end{array}\right], \quad D_{12}=\left[\begin{array}{cc}
0.5 & 0.8 \\
-0.1 & -0.4
\end{array}\right], \\
D_{21}=\left[\begin{array}{cc}
0.2 & 0.3 \\
-0.2 & -0.3
\end{array}\right], \quad D_{22}=\left[\begin{array}{cc}
0.2 & 0.4 \\
0.1 & 0.4
\end{array}\right] .
\end{gathered}
$$

The fuzzy sets of " $M_{11}^{1}, M_{11}^{2}, M_{21}^{1}$, and $M_{21}^{2}$ " are represented by the following membership functions, respectively.

$$
\begin{gathered}
M_{11}^{1}\left(x_{2}(t)\right)=h_{11}\left(x_{2}(t)\right)=1-\frac{1}{1+e^{-2 x_{2}(t)}}, \quad M_{11}^{2}\left(x_{2}(t)\right)=h_{12}\left(x_{2}(t)\right)=\frac{1}{1+e^{-2 x_{2}(t)}}, \\
M_{21}^{1}\left(x_{2}(t)\right)=h_{21}\left(x_{2}(t)\right)=1-\frac{1}{1+e^{\left(-2\left(x_{2}(t)-0.3\right)\right)},} \quad M_{21}^{2}\left(x_{2}(t)\right)=h_{22}\left(x_{2}(t)\right)=\frac{1}{1+e^{\left(-2\left(x_{2}(t)-0.3\right)\right)}} .
\end{gathered}
$$




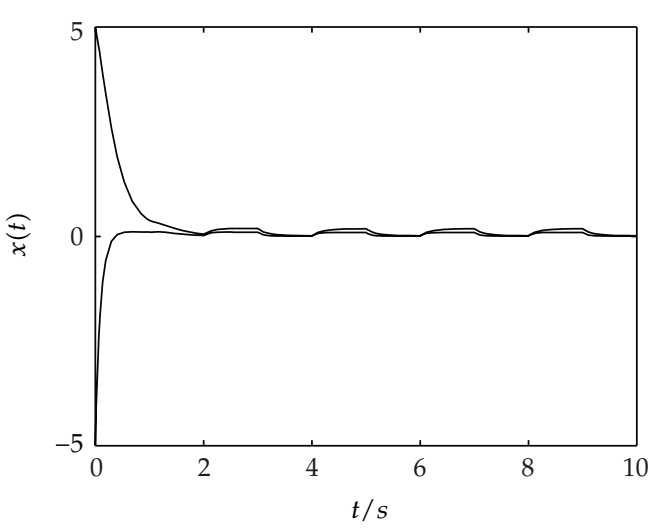

(a)

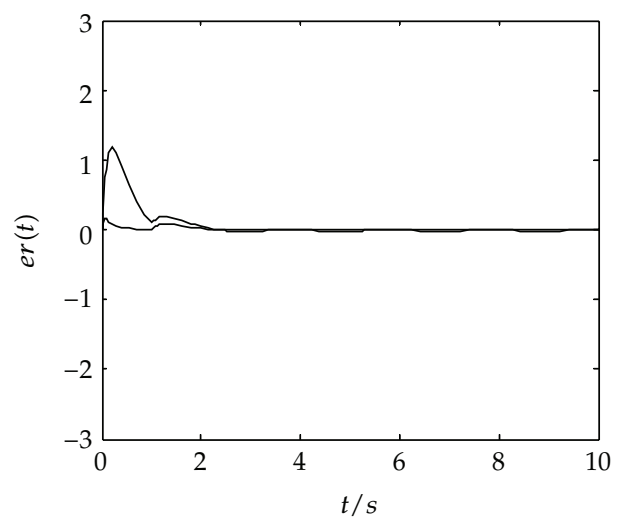

(b)

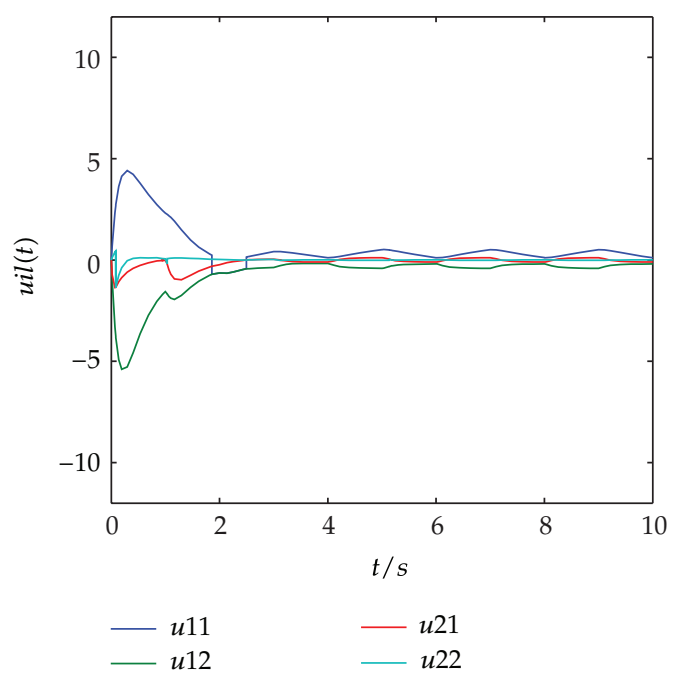

(c)

Figure 3: (a) Response evolution of system state variables with the fast switching controller. (b) Response evolution of system error variables with the fast switching controller. (c) Control signal of the fast switching controller.

Choosing $\lambda_{i j_{i}}=1, j_{i}, \vartheta_{i}=1,2, i=1,2$ for (4.33), we can have the matrix

$$
\begin{gathered}
R=0.1006, \quad P_{11}=\left[\begin{array}{ll}
1.3092 & 0.7438 \\
0.7438 & 3.1293
\end{array}\right], \quad P_{22}=\left[\begin{array}{ll}
1.6276 & 0.0062 \\
0.0062 & 2.4938
\end{array}\right], \\
S_{11}=\left[\begin{array}{cc}
17.7120 & 0.1379 \\
0.1379 & 18.1660
\end{array}\right], \quad S_{22}=\left[\begin{array}{cc}
15.0209 & -0.0077 \\
-0.0077 & 8.2345
\end{array}\right] .
\end{gathered}
$$

Taking the initial condition as $x(0)=[-5,5]^{T}$, with $\tau=1, r(t)$ and $\omega(t)$ are generated by pulse wave form. The simulation result for the fast switching controller of SF time-delay system is depicted in Figure 3. 


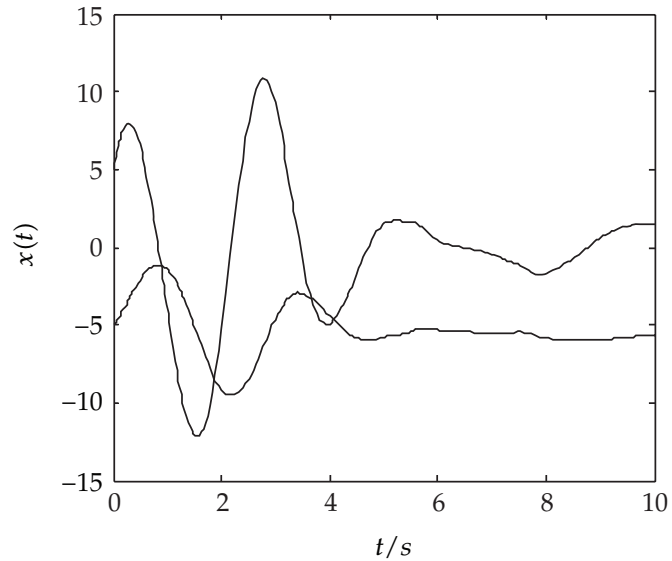

(a)

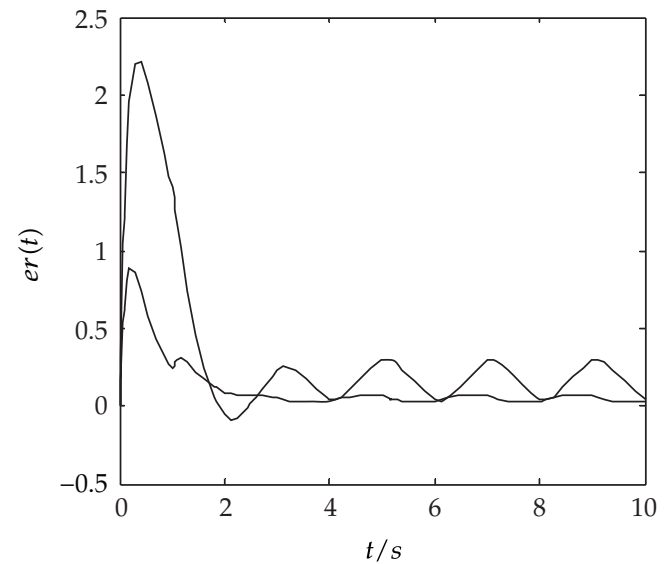

(b)

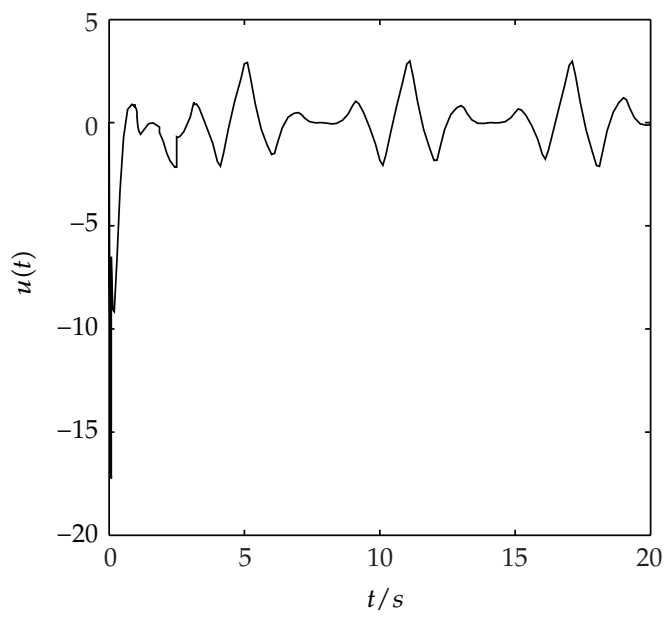

(c)

Figure 4: (a) Response evolution of system state variables with the traditional PDC controller. (b) Response evolution of system error variables with the traditional PDC controller. (c) Control signal of the traditional PDC fuzzy controller.

To investigate the effectiveness of the proposed fast switching controller of the SF system here, we now compare the traditional PDC controller [21-23, 36]. Obviously, the global control of the traditional PDC fuzzy controller is

$$
u(t)=\sum_{i=1}^{N_{i}} \sum_{l=1}^{N_{i}} v_{i} h_{i l} K_{i l} e(t)
$$

The state feedback gains of subsystems are obtained as

$$
\begin{gathered}
K_{11}=\left[\begin{array}{ll}
-0.332 & -0.145
\end{array}\right], \quad K_{12}=\left[\begin{array}{ll}
-0.0523 & -5.573
\end{array}\right], \\
K_{21}=\left[\begin{array}{ll}
-2.3 & -4.12
\end{array}\right], \quad K_{22}=\left[\begin{array}{ll}
-2.34 & -4.1
\end{array}\right] .
\end{gathered}
$$


For the same data and the same initial condition $x(0)=[-5,5]^{T}$, the simulation result is depicted in Figure 4. It should be noted that the fast switching controller can improve the transient characteristic. Figures 3 and 4 indicate that the proposed method gives better results in convergence.

\section{Conclusion}

In this paper, tracking control for SF systems with time delay is investigated. We use single Lyapunov function technique and a switching law to design a tracking control law such that the $H_{\infty}$ model reference tracking performance is satisfied. The fast switching controller design problem can be solved efficiently by using linear matrix inequalities and convex optimization techniques. Simulation example shows the validity of the switching control law.

\section{Acknowledgments}

This work is supported by National Nature Science Foundation under Grant 61004039, the Science Foundation of Educational Department of Liaoning Province under Grant 2010375, and Excellent Talented Person Foundation of Educational Department of Liaoning Province under Grant LJQ2011127. The authors also gratefully acknowledge the helpful comments and suggestions of the reviewers, which have improved the presentation.

\section{References}

[1] D. Z. Cheng, L. Guo, Y. D. Lin, and Y. Wang, "Stabilization of switched linear systems," IEEE Transactions on Automatic Control, vol. 50, no. 5, pp. 661-666, 2005.

[2] J. Zhao and G. M. Dimirovski, "Quadratic stability of a class of switched nonlinear systems," IEEE Transactions on Automatic Control, vol. 49, no. 4, pp. 574-578, 2004.

[3] G. M. Xie and L. Wang, "Necessary and sufficient conditions for controllability and observability of switched impulsive control systems," IEEE Transactions on Automatic Control, vol. 49, no. 6, pp. 960 966, 2004.

[4] J. Liu, X. Z. Liu, and W. C. Xie, "Input-to-state stability of impulsive and switching hybrid systems with time-delay," Automatica, vol. 47, no. 5, pp. 899-908, 2011.

[5] R. Wang, G. P. Liu, W. Wang, D. Rees, and Y. B. Zhao, " $\mathrm{H}_{\infty}$ control for networked predictive control systems based on the switched Lyapunov function method," IEEE Transactions on Industrial Electronics, vol. 57, no. 10, pp. 3565-3571, 2010.

[6] M. Wicks, P. Peleties, and R. Decarlo, "Switched controller synthesis for the quadratic stabilisation of a pair of unstable linear systems," European Journal of Control, vol. 4, no. 2, pp. 140-147, 1998.

[7] L. Dugard and E. L. Verrist, Stability and Control of Time-Delay Systems, Springer, New York, NY, USA, 1998.

[8] E. Fridman and U. Shaked, "A descriptor system approach to $\mathrm{H}_{\infty}$ control of linear time-delay systems," IEEE Transactions on Automatic Control, vol. 47, no. 2, pp. 253-270, 2002.

[9] Q. L. Han and K. Gu, "On robust stability of time-delay systems with norm-bounded uncertainty," IEEE Transactions on Automatic Control, vol. 46, no. 9, pp. 1426-1431, 2001.

[10] Y. He, M. Wu, J. H. She, and G. P. Liu, "Parameter-dependent Lyapunov functional for stability of time-delay systems with polytopic-type uncertainties," IEEE Transactions on Automatic Control, vol. 49 , no. 5, pp. 828-832, 2004.

[11] J. P. Richard, "Time-delay systems: an overview of some recent advances and open problems," Automatica, vol. 39, no. 10, pp. 1667-1694, 2003.

[12] S. Kim, S. A. Campbell, and X. Liu, "Stability of a class of linear switching systems with time delay," IEEE Transactions on Circuits and Systems I, vol. 53, no. 2, pp. 384-393, 2006. 
[13] X. M. Sun, J. Zhao, and D. J. Hill, "Stability and $L_{2}$-gain analysis for switched delay systems: a delaydependent method," Automatica, vol. 42, no. 10, pp. 1769-1774, 2006.

[14] D. Xie and X. Chen, "Observer-based switched control design for switched linear systems with time delay in detection of switching signal," IET Control Theory and Applications, vol. 2, no. 5, pp. 437-445, 2008.

[15] G. Xie and L. Wang, "Stabilization of switched linear systems with time-delay in detection of switching signal," Journal of Mathematical Analysis and Applications, vol. 305, no. 1, pp. 277-290, 2005.

[16] D. S. Du, B. Jiang, P. Shi, and S. S. Zhou, "H $\mathrm{H}_{\infty}$ filtering of discrete-time switched systems with state delays via switched Lyapunov function approach," IEEE Transactions on Automatic Control, vol. 52, no. 8, pp. 1520-1525, 2007.

[17] Q. K. Li, J. Zhao, and G. M. Dimirovski, "Robust tracking control for switched linear systems with time-varying delays," IET Control Theory and Applications, vol. 2, no. 6, pp. 449-457, 2008.

[18] H. K. Lam and F. H. F. Leung, "Fuzzy rule-based combination of linear and switching state-feedback controllers," Fuzzy Sets and Systems, vol. 156, no. 2, pp. 153-184, 2005.

[19] W. J. Wang and C. H. Sun, "Relaxed stability and stabilization conditions for a T-S fuzzy discrete system," Fuzzy Sets and Systems, vol. 156, no. 2, pp. 208-225, 2005.

[20] R. Palm and D. Driankov, "Fuzzy switched hybrid systems-modeling and identification," in Proceedings of IEEE International Symposium on Computational Intelligence in Robotics and Automation (CIRA), Intelligent Systems and Semiotics (ISAS), and Intelligent Control (ISIC), pp. 130-135, September 1998.

[21] K. Tanaka, M. Iwasaki, and H. O. Wang, "Switching control of an R/C hovercraft: stabilization and smooth switching," IEEE Transactions on Systems, Man, and Cybernetics B, vol. 31, no. 6, pp. 853-863, 2001.

[22] K. Tanaka, M. Iwasaki, and H. O. Wang, "Stability and smoothness conditions for switching fuzzy systems," in Proceedings of the American Control Conference, pp. 2474-2478, June 2000.

[23] K. Tanaka, M. Iwasaki, and H. O. Wang, "Stable switching fuzzy control and its application to a Hovercraft type vehicle," in Proceedings of the 9th IEEE International Conference on Fuzzy Systems, pp. 804-809, May 2000.

[24] W. J. Wang, Y. J. Chen, and C. H. Sun, "Relaxed stabilization criteria for discrete-time T-S fuzzy control systems based on a switching fuzzy model and piecewise Lyapunov function," IEEE Transactions on Systems, Man, and Cybernetics B, vol. 37, no. 3, pp. 551-559, 2007.

[25] D. J. Choi, S. S. Lee, and P. Park, “Output-feedback $\mathrm{H}_{\infty}$ control of discrete-time switching fuzzy systems," in Proceedings of the 12th IEEE International Conference on Fuzzy Systems (FUZZ '03), vol. 1, pp. 441-446, May 2003.

[26] D. J. Choi and P. Park, "Guaranteed cost controller design for discrete-time switching fuzzy systems," IEEE Transactions on Systems, Man, and Cybernetics B, vol. 34, no. 1, pp. 110-119, 2004.

[27] H. Yang, H. Liu, G. M. Dimirovski, and J. Zhao, "Stabilization control for a class of switched fuzzy discrete-time systems," in Proceedings of IEEE International Conference on Fuzzy Systems, pp. 1345-1350, July 2007.

[28] Y. M. Li, Y. Yang, and L. Li, "Adaptive backstepping fuzzy control based on type 2- fuzzy system," Journal of Applied Mathematics, vol. 2012, Article ID 658424, 27 pages, 2012.

[29] A. V. Savkin and A. S. Matveev, "Cyclic linear differential automata: a simple class of hybrid dynamical systems," Automatica, vol. 36, no. 5, pp. 727-734, 2000.

[30] M. Sugeno, M. F. Griffin, and A. Bastian, "Fuzzy hierarchical control of an unmanned helicopter," in Proceedings of the 5th International Fuzzy Systems and Applications Conference (IFSA '93), pp. 1262-1265, July 1993.

[31] Q. K. Li, J. Zhao, G. M. Dimirovski, and X. J. Liu, “Tracking control for switched linear systems with time-delay: a state-dependent switching method," Asian Journal of Control, vol. 11, no. 5, pp. 517-526, 2009.

[32] B. Chen, M. Wang, X. P. Liu, and P. Shi, "Adaptive fuzzy tracking control for a class of perturbed strict-feedback nonlinear time-delay systems," Fuzzy Sets and Systems, vol. 159, no. 8, pp. 949-967, 2008.

[33] P. Liu and T. S. Chiang, " $\mathrm{H}_{\infty}$ output tracking fuzzy control for nonlinear systems with time-varying delay," Applied Soft Computing, vol. 12, no. 9, pp. 2963-2972, 2012.

[34] T. S. Chiang and P. Liu, "Robust output tracking control for discrete-time nonlinear systems with time-varying delay: virtual fuzzy model LMI-based approach," Expert Systems with Applications, vol. 39, no. 9, pp. 8239-8247, 2012. 
[35] L. Zhang, H. Yang, and Y. W. Jing, "Adaptive robust tracking control for a class of uncertain switched fuzzy systems," Journal of Northeastern University, vol. 29, no. 8, pp. 1073-1076, 2008.

[36] Y. Liu, G. M. Dimirovski, and J. Zhao, "Robust output feedback control for a class of uncertain switching fuzzy systems," in Proceedings of the 17th IFAC World Congress (IFAC '08), pp. 4773-4778, July 2008.

[37] S. C. Tong, T. Wang, and H. X. Li, "Fuzzy robust tracking control for uncertain nonlinear systems," International Journal of Approximate Reasoning, vol. 30, no. 2, pp. 73-90, 2002.

[38] H. O. Wang, K. Tanaka, and M. F. Griffin, "An approach to fuzzy control of nonlinear systems: stability and design issues," IEEE Transactions on Fuzzy Systems, vol. 4, no. 1, pp. 14-23, 1996. 


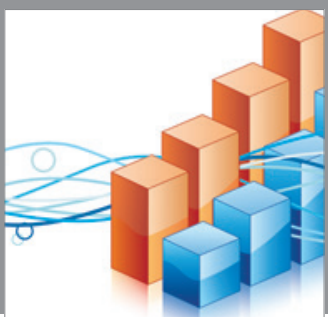

Advances in

Operations Research

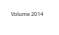

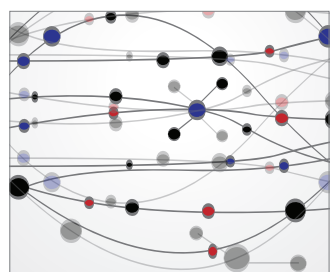

\section{The Scientific} World Journal
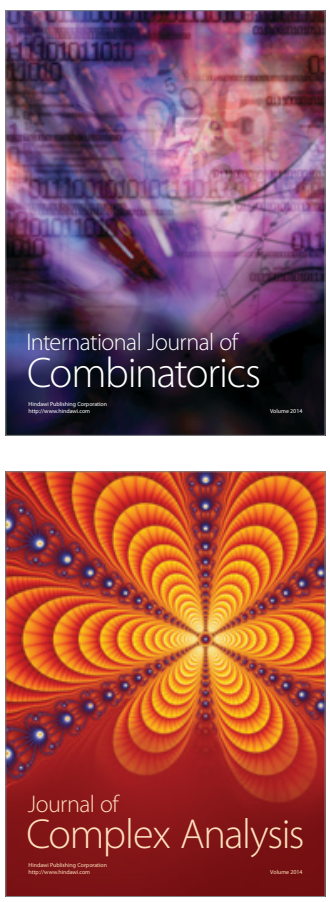

International Journal of

Mathematics and

Mathematical

Sciences
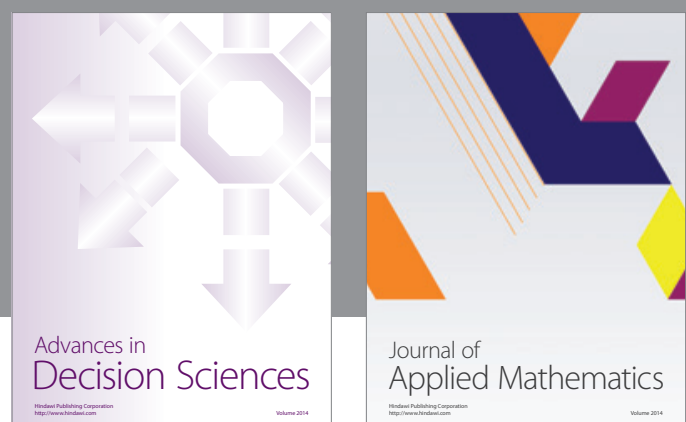

Journal of

Applied Mathematics
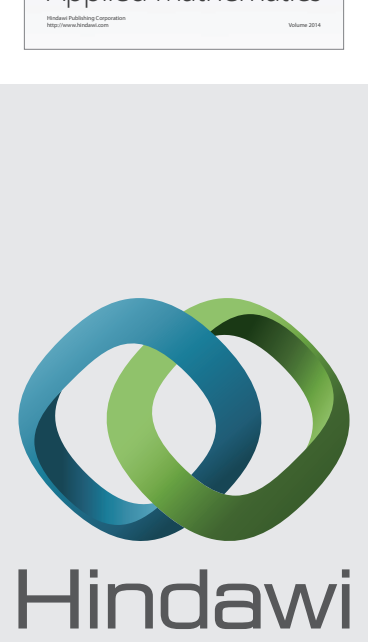

Submit your manuscripts at http://www.hindawi.com
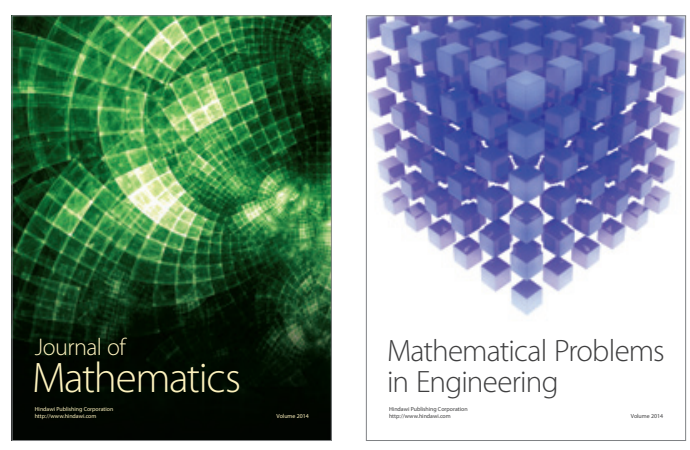

Mathematical Problems in Engineering
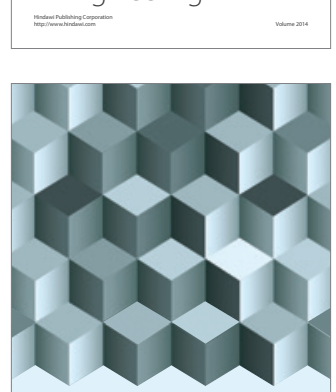

Journal of

Function Spaces
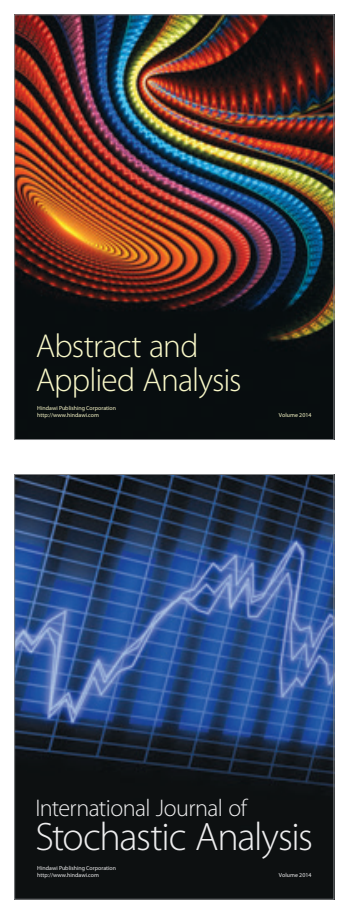

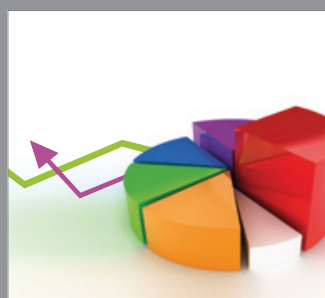

ournal of

Probability and Statistics

Promensencen
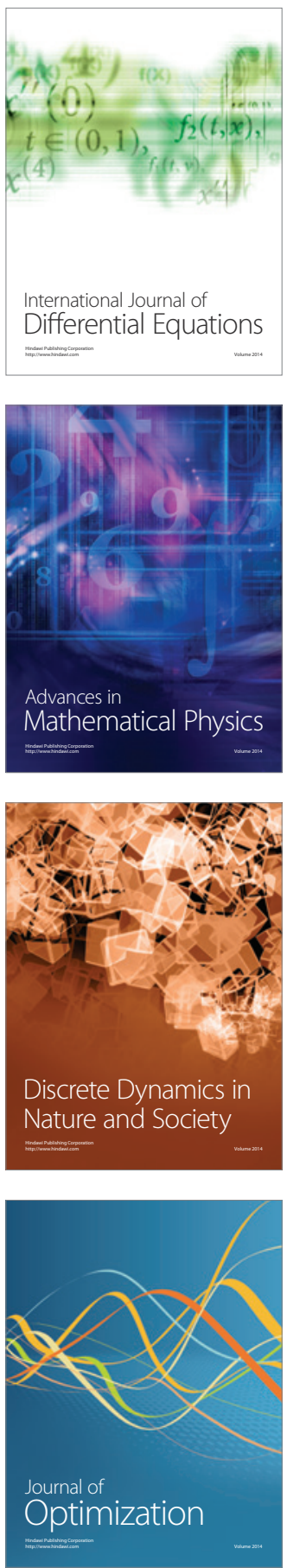